\title{
Skin Sloughing
}

National Cancer Institute

\section{Source}

National Cancer Institute. Skin Sloughing. NCI Thesaurus. Code C112110.

Peeling or shedding of skin in sheets. 